\title{
Solid-Liquid Reaction in the System $\mathrm{Si}_{3} \mathrm{~N}_{4}-\mathrm{Y}_{3} \mathrm{Al}_{5} \mathrm{O}_{12}-\mathrm{Y}_{2} \mathrm{Si}_{2} \mathrm{O}_{7}$ under $1 \mathrm{MPa}$ of Nitrogen
}

\author{
Zhen-Kun Huang and Tseng-Ying Tien*
}

Department of Materials Science and Engineering, The University of Michigan, Ann Arbor, Michigan 48109

\begin{abstract}
The melting behaviors of selected compositions in the system $\mathrm{Si}_{3} \mathrm{~N}_{4}-\mathrm{Y}_{3} \mathrm{Al}_{5} \mathrm{O}_{12}-\mathrm{Y}_{2} \mathrm{Si}_{2} \mathrm{O}_{7}$ were determined under $1 \mathrm{MPa}$ of nitrogen. The behavior phase diagrams of the ternary and their binary systems are presented. The ternary eutectic composition contains 10 wt $\% \mathrm{Si}_{3} \mathrm{~N}_{4}, 27$ wt $\% \mathrm{Y}_{3} \mathrm{Al}_{5} \mathrm{O}_{12}$, and $63 \mathrm{wt} \% \mathrm{Y}_{2} \mathrm{Si}_{2} \mathrm{O}_{7}$ with a eutectic temperature of $1430^{\circ} \mathrm{C}$. The binary eutectic compositions and temperatures are 25 wt $\% \mathrm{Si}_{3} \mathrm{~N}_{4}$ and $75 \mathrm{wt} \% \mathrm{Y}_{3} \mathrm{Al}_{5} \mathrm{O}_{12}$ at $1650^{\circ} \mathrm{C}, 10 \mathrm{wt} \% \mathrm{Si}_{3} \mathrm{~N}_{4}$ and $90 \mathrm{wt} \% \quad \mathrm{Y}_{2} \mathrm{Si}_{2} \mathrm{O}_{7}$ at $1570^{\circ} \mathrm{C}$, and $35 \mathrm{wt} \% \mathrm{Y}_{3} \mathrm{Al}_{5} \mathrm{O}_{12}$ and $65 \mathrm{wt} \% \mathrm{Y}_{2} \mathrm{Si}_{2} \mathrm{O}_{7}$ at $1520^{\circ} \mathrm{C}$.
\end{abstract}

\section{Introduction}

Qilicon nitride ceramics are usually densified in the presence of a reactive liquid. The liquid is normally comprised of the eutectic melt from the surface layer of the silicon nitride powder and the oxide sintering additives used. The presence of the liquid also aids the microstructural development which gives silicon nitride ceramics their superior mechanical properties.

The most common oxide sintering additives for $\mathrm{Si}_{3} \mathrm{~N}_{4}$ are $\mathrm{Y}_{2} \mathrm{O}_{3}$ and $\mathrm{AI}_{2} \mathrm{O}_{3}$. Phase equilibria have been studied extensively in silicon nitride-metal oxide systems. Subsolidus phase equilibria have been reported in the systems $\mathrm{Si}, \mathrm{Al} / \mathrm{N}, \mathrm{O}^{\prime}$ and $\mathrm{Si}, \mathrm{Y} / \mathrm{N}, \mathrm{O} .^{2}$ The solid-liquid isotherm at $1750^{\circ} \mathrm{C}$ in the system $\mathrm{Si}, \mathrm{Al} / \mathrm{N}, \mathrm{O}^{3}$ has been studied. Combining the systems $\mathrm{Si}, \mathrm{Al} /$ $\mathrm{N}, \mathrm{O}$ and $\mathrm{Si}, \mathrm{Y} / \mathrm{N}, \mathrm{O}$ gives the quasi-quaternary system $\mathrm{Si}, \mathrm{Al}, \mathrm{Y} /$ N,O. ${ }^{4,5}$ The system Si,Al,Y/N,O is composed of 68 compatibility tetrahedra, but the most promising compositions for hightemperature ceramics are bounded by the $\mathrm{Si}_{3} \mathrm{~N}_{4}-\mathrm{Al}_{2} \mathrm{O}_{3}$ : $\mathrm{AIN}$ $\mathrm{YAG}-\mathrm{Y}_{2} \mathrm{Si}_{2} \mathrm{O}_{7}$ compatibility tetrahedron. ${ }^{5} \mathrm{YAG}$ refers to yttrium aluminum garnet, $3 \mathrm{Y}_{2} \mathrm{O}_{3} \cdot 5 \mathrm{Al}_{2} \mathrm{O}_{3} \cdot \mathrm{Si}_{3} \mathrm{~N}_{4}$ ceramics with YAG as a second phase have been reported by Lewis et al. ${ }^{6}$ However, Hohnke and Tien ${ }^{7}$ were the first to study the solidliquid reactions in the $\mathrm{Si}_{3} \mathrm{~N}_{4}-\beta_{60}-\mathrm{YAG}$ region. They found a two-phase field over the entire $\mathrm{Si}_{3} \mathrm{~N}_{4}-\beta_{61}-\mathrm{YAG}$ plane at $1550^{\circ} \mathrm{C}$, with the liquid first forming along the $\mathrm{YAG}-\beta_{60}$ join. $\beta_{60}$ refers to the 60 equiv $\%$ Al-O substituted $\beta$-silicon nitride with the formula $\mathrm{Si}_{6-x} \mathrm{Al}_{x} \mathrm{O}_{x} \mathrm{~N}_{8-x}$ with $x=4 ; 1650^{\circ}$ and $1750^{\circ} \mathrm{C}$ isotherms in this system have also been studied by Wisnudel and Tien. ${ }^{8}$

The focus of this research is to clarify the solid-liquid reactions in the system $\mathrm{Si}_{3} \mathrm{~N}_{4}-\mathrm{YAG}-\mathrm{Y}_{2} \mathrm{Si}_{2} \mathrm{O}_{7}$.

\section{Experimental Procedure and Results}

The starting powders used were $\mathrm{Si}_{3} \mathrm{~N}_{4}$ (UBE-SN-E10, Japan), $\mathrm{Al}_{2} \mathrm{O}_{3}$ (AKP-50, Sumitomo Chemical Co. Ltd., Japan), $\mathrm{Y}_{2} \mathrm{O}_{3}(99.99 \%$, Aldrich Chemical Co. Inc., Milwaukee, WI),

\footnotetext{
S. Danforth contributing editor
}

\footnotetext{
Manuscript No. 193875. Received January 26, 1994; approved July 6, 1994

Supported by the Ceramic Technology Project, Department of Energy Office of Transportation Technologies, under Coniract No. DE-AC05-84OR21400 with Martin Marietta Energy Sysiems, Inc.
}

*Member, American Ceramic Socicly. and $\mathrm{SiO}_{2}$ (Aerosil 380, Degussa Co., U.S.A.). More than 50 compositions were prepared in the system, among which the smallest interval of some compositions was $5 \mathrm{wt} \%$. The starting powders of different compositions were weighed and mixed in an agate mortar and pestle under isopropyl alcohol for $1.5 \mathrm{~h}$. The mixtures were dried and pressed into pellets $(0.25 \mathrm{~cm} \times$ $1 \mathrm{~cm}$ in diameter) and then isostatically pressed under $300 \mathrm{MPa}$. The pellets were placed on a BN powder-bed in a graphite die with a screw cap and were sintered in a graphite furnace under $1 \mathrm{MPa}$ of nitrogen at different temperatures for $2 \mathrm{~h}$. The natural cooling rate of the samples after sintering was $40^{\circ} \mathrm{C} / \mathrm{min}$ from power-off until $1000^{\circ} \mathrm{C}$. The weight loss of the samples was measured by weighing the samples before and after sintering.

$\mathrm{X}$-ray diffraction was used for phase identification. Scanning electron micrographs from polished surfaces were used to quantify the amount of liquid phase present in the samples." The liquid phase was crystallized after densification to verify the location of the starting composition.

Melting behaviors of some compositions were determined by visual observation. The level rule was used to determine the liquidus location. The data points collected are listed in Tables I through IV. These data were used to construct the phase diagrams in Figs. 1, 2, and 3.

\section{Discussion}

\section{(1) The Binary System $\mathrm{Si}_{3} \mathrm{~N}_{4}-\mathrm{Y}_{3} \mathrm{Al}_{5} \mathrm{O}_{12}$}

Previous reports ${ }^{8}$ indicate that $\mathrm{Si}_{3} \mathrm{~N}_{4}$ and $\mathrm{Y}_{3} \mathrm{Al}_{5} \mathrm{O}_{12}$ form a binary join at $1550^{\circ} \mathrm{C}$ under flowing nitrogen conditions. At higher temperatures, a liquid phase is observed, and this system forms a true binary join.

In the present work on this binary system, the experimental results indicate that silicon nitride could be compatible with YAG up to a higher temperature $\left(1630^{\circ} \mathrm{C}\right)$ under $1 \mathrm{MPa}$ of nitrogen. The eutectic point in this binary system is at $1650^{\circ} \mathrm{C}$ with a composition of $25 \mathrm{wt} \% \mathrm{Si}_{3} \mathrm{~N}_{4}$ and $75 \mathrm{wt} \% \mathrm{Y}_{2} \mathrm{Al}_{5} \mathrm{O}_{12}$. At $1650^{\circ} \mathrm{C}$ the weight losses for the compositions investigated in this system were small (below $2 \mathrm{wt} \%$ ). Figure 1 shows the behavior phase diagram of the $\mathrm{Si}_{3} \mathrm{~N}_{4}-\mathrm{Y}_{3} \mathrm{Al}_{5} \mathrm{O}_{12}$ system under $1 \mathrm{MPa}$ of nitrogen. The weight loss became greater at higher temperatures. Up to $10 \mathrm{wt} \%$ weight loss was observed at $1800^{\circ} \mathrm{C}$.

$\mathrm{Si}_{3} \mathrm{~N}_{4}$-rich samples with higher weight loss showed three phases: $\mathrm{Si}_{3} \mathrm{~N}_{4}$, monoclinic $\mathrm{J}_{\mathrm{ss}}$, and liquid. $\mathrm{J}_{\mathrm{ss}}$ has a higher melting point $\left(2000^{\circ} \mathrm{C}\right)$. Higher temperature and more oxygen-rich compositions favor the formation of $J_{\mathrm{ss}}$ phase.

\section{(2) The Binary System $\mathrm{Si}_{3} \mathrm{~N}_{4}-\mathrm{Y}_{2} \mathrm{Si}_{2} \mathrm{O}_{7}$}

The system $\mathrm{Si}_{3} \mathrm{~N}_{4}-\mathrm{Y}_{2} \mathrm{Si}_{2} \mathrm{O}_{7}$ is a true binary. The specimens have high weight loss. The hexagonal $\mathrm{H}$-phase $\mathrm{Y}_{5}\left(\mathrm{SiO}_{4}\right)_{3} \mathrm{~N}$ often appears. The binary composition of $10 \mathrm{wt} \% \mathrm{Si}_{3} \mathrm{~N}_{4}: 90 \mathrm{wt} \%$ $\mathrm{Y}_{2} \mathrm{Si}_{2} \mathrm{O}_{7}$ is the eutectic which melts at $1570^{\circ} \mathrm{C}$.

\section{(3) The Binary System $\mathrm{Y}_{3} \mathrm{Al}_{5} \mathrm{O}_{12}-\mathrm{Y}_{2} \mathrm{Si}_{2} \mathrm{O}_{7}$}

The lowest melting composition in this binary system was found to contain $35 \mathrm{wt} \% \quad \mathrm{Y}_{3} \mathrm{Al}_{5} \mathrm{O}_{12}: 65 \mathrm{wt} \% \quad \mathrm{Y}_{2} \mathrm{Si}_{2} \mathrm{O}_{7}$ with a eutectic temperature at $1520^{\circ} \mathrm{C}$. No weight loss was detected for 
Table I. Compositions, Firing Conditions, Melting Behaviors, and Crystal Phase Analyses in the System $\mathrm{Si}_{3} \mathrm{~N}_{4}-\mathrm{Y}_{3} \mathrm{Al}_{5} \mathrm{O}_{12}$ under $1 \mathrm{MPa}$ of Nitrogen

\begin{tabular}{|c|c|c|c|c|c|}
\hline & $\begin{array}{c}\mathrm{Si}_{3} \mathrm{~N}_{4}: \mathrm{Y}_{3} \mathrm{Al}_{5} \mathrm{O}_{12} \\
\text { composition (wt\%) }\end{array}$ & $\begin{array}{l}\text { Temp } \\
\left({ }^{\circ} \mathrm{C}\right)\end{array}$ & $\begin{array}{l}\text { Wt loss } \\
(w t \%)\end{array}$ & Melting behaviors & Phase analyses* \\
\hline \multirow[t]{4}{*}{$(1)$} & $100: 0$ & 1900 & 6.1 & Not densified & $\alpha-\mathrm{SN}, \beta-\mathrm{SN}$ \\
\hline & & 1800 & 2.1 & Not densified & $\alpha-\mathrm{SN}, \beta-\mathrm{SN}$ \\
\hline & & 1750 & 2.0 & Not densified & \\
\hline & & 1650 & 1.2 & No melting & \\
\hline \multirow[t]{6}{*}{ (2) } & $80: 20$ & 1900 & 19.7 & Large shrinkage & \\
\hline & & 1800 & 5.4 & Densified & $\mathrm{SN}, \mathbf{J}_{\mathrm{s}}$ \\
\hline & & 1750 & 2.0 & Densified & \\
\hline & & 1700 & 0.7 & Densified & SN, B \\
\hline & & 1650 & 0.3 & Densified & $\mathrm{SN}, \mathrm{G}, \mathrm{H}$ \\
\hline & & 1550 & 1.0 & Not melted & $\mathrm{SN}, \mathrm{G}$ \\
\hline \multirow[t]{5}{*}{$(3)$} & $70: 30$ & 1900 & 15.9 & Large shrinkage & $\mathrm{SN}, \mathrm{J}$ \\
\hline & & 1800 & 5.9 & Densified & $\mathrm{SN}, \mathrm{J}_{\mathrm{s}}$ \\
\hline & & 1750 & 3.0 & Densified & $\mathrm{SN}, \mathbf{J}_{\mathrm{ss}}$ \\
\hline & & 1700 & 1.0 & Densified & SN, B \\
\hline & & 1650 & 1.0 & Densified & \\
\hline \multirow[t]{5}{*}{ (4) } & $60: 40$ & 1900 & 27.6 & Large shrinkage & \\
\hline & & 1800 & 6.2 & Densified & $\mathrm{SN}, \mathrm{J}_{\mathrm{ss}}$ \\
\hline & & 1750 & 4.1 & Densified & $S N, J_{\mathrm{ss}}$ \\
\hline & & 1700 & 2.1 & Densified & $\mathrm{SN}, \mathrm{B}, \mathrm{J}$ \\
\hline & & 1650 & 0.8 & Densified & \\
\hline \multirow[t]{5}{*}{ (5) } & $50: 50$ & 1900 & 16.9 & Partly melted & \\
\hline & & 1800 & 8.8 & Partly melted & $J_{i s}, \mathrm{SN}$ \\
\hline & & 1750 & 5.2 & Partly melted & \\
\hline & & 1700 & 3.7 & Partly melted & $\mathrm{J}_{4 \mathrm{~s}}, \mathrm{SN}$ \\
\hline & & 1650 & 1.8 & Densified & \\
\hline \multirow[t]{4}{*}{ (6) } & $40: 60$ & 1800 & 15.5 & Partly melted & \\
\hline & & 1750 & 8.2 & Partly melted & $\mathrm{B}, \mathrm{J}_{\mathrm{s},}, \mathrm{SN}$ \\
\hline & & 1700 & 3.9 & Partly melted & $\mathrm{J}, \mathrm{SN}$ \\
\hline & & 1650 & 0.7 & Partly melted & \\
\hline \multirow[t]{5}{*}{ (7) } & $30: 70$ & 1800 & 9.8 & Melted (bubbles) & \\
\hline & & 1750 & 4.6 & Melted (bubbles) & \\
\hline & & 1650 & & Partly melted & $\mathrm{SN}$ \\
\hline & & 1630 & 1.5 & No melting & \\
\hline & & 1600 & 1.9 & No melting & $\mathrm{G}, \mathrm{SN}$ \\
\hline \multirow[t]{3}{*}{ (8) } & $25: 75$ & 1650 & 1.7 & Melted (bubbles) & $\mathrm{SN}, \mathrm{G}$ \\
\hline & & 1630 & 0.9 & No melting & \\
\hline & & 1600 & 0.9 & No melting & $\mathrm{G}, \mathrm{SN}$ \\
\hline \multirow[t]{6}{*}{ (9) } & $20: 80$ & 1800 & 6.0 & Melted (bubbles) & \\
\hline & & 1750 & 2.4 & Melted (bubbles) & $\mathrm{G}, \mathbf{J}_{\mathrm{w}}$ \\
\hline & & 1650 & 2.0 & Mostly melted & $\mathrm{G}$ \\
\hline & & 1630 & 2.3 & Partly melted & \\
\hline & & 1600 & 1.2 & No melting & $\mathrm{G}, \mathrm{SN}$ \\
\hline & & 1550 & 1.1 & No melting & G, SN \\
\hline \multirow[t]{3}{*}{$(10)$} & $10: 90$ & 1800 & 3.3 & Melted & \\
\hline & & 1750 & 2.0 & Melted (bubbles) & \\
\hline & & 1650 & & Slightly melted & \\
\hline
\end{tabular}

$* \mathrm{SN}=\mathrm{Si}_{3} \mathrm{~N}_{4}\left(\alpha\right.$ and $/$ or $\beta$ ), $\mathrm{G}=\mathrm{Y}_{3} \mathrm{Al}_{5} \mathrm{O}_{12}(\mathrm{YAG}$ or garnet $), \mathrm{B}=\mathrm{Y}_{2} \mathrm{SiAlO}_{5} \mathrm{~N}$ (B-phase), $\mathrm{J}_{v 4}=$ solid solution of $2 \mathrm{Y}_{2} \mathrm{O}_{7} \cdot \mathrm{Si}_{2} \mathrm{~N}_{2} \mathrm{O}$ and $2 \mathrm{Y}_{2} \mathrm{O}_{4} \cdot \mathrm{Al}_{2} \mathrm{O}_{3}, \mathrm{H}=\mathrm{Y}_{4}\left(\mathrm{SiO}_{4}\right)_{3} \mathrm{~N}$ (H-phase).

Table II. Compositions, Firing Conditions, Melting Behaviors, and Crystal Phase Analyses in the System $\mathrm{Si}_{3} \mathrm{~N}_{4}-\mathrm{Y}_{2} \mathrm{Si}_{2} \mathrm{O}_{7}$

\begin{tabular}{|c|c|c|c|c|c|}
\hline & $\begin{array}{c}\mathrm{Si}_{3} \mathrm{~N}_{4}: \mathrm{Y}_{2} \mathrm{Si}_{2} \mathrm{O}_{7} \\
\text { composition (wt\%) }\end{array}$ & $\begin{array}{l}\text { Temp } \\
\left({ }^{\circ} \mathrm{C}\right)\end{array}$ & $\begin{array}{l}\text { Wt loss } \\
(w t \%)\end{array}$ & Melting behaviors & Phase analyses* \\
\hline \multirow[t]{2}{*}{ (11) } & $80: 20$ & 1650 & 4.7 & Densified & \\
\hline & & 1550 & 2.0 & Not melted & $\mathrm{SN}, \mathrm{H}$ \\
\hline$(12)$ & $70: 30$ & 1650 & 6.8 & Densified & $\mathrm{SN}, \mathrm{H}$ \\
\hline (13) & $60: 40$ & 1650 & 4.1 & Densified & $\mathrm{SN}, \mathrm{H}$ \\
\hline (14) & $50: 50$ & 1650 & 5.1 & Densified & $\mathrm{H}, \mathrm{SN}$ \\
\hline$(15)$ & $40: 60$ & 1650 & 6.4 & Slightly melted & $\mathrm{H}, \mathrm{SN}, \mathrm{YS}$ \\
\hline \multirow[t]{3}{*}{ (16) } & $30: 70$ & 1650 & & Melted (bubbles) & $\mathrm{YS}, \mathrm{H}, \mathrm{SN}$ \\
\hline & & 1550 & 3.4 & No melting & \\
\hline & & 1500 & 2.2 & No melting & \\
\hline \multirow[t]{2}{*}{ (17) } & $20: 80$ & 1650 & 12.0 & Mejted & YS, SN, H \\
\hline & & 1550 & & No melting & \\
\hline (18) & $15: 85$ & 1600 & 3.4 & Melted (bubbles) & \\
\hline \multirow[t]{3}{*}{ (19) } & $10: 90$ & 1650 & 9.3 & Melted & \\
\hline & & 1600 & 4.1 & Melted (bubbles) & \\
\hline & & 1550 & 1.2 & No melting & YS, SN \\
\hline \multirow[t]{2}{*}{$(20)$} & $05: 95$ & 1650 & 4.8 & Melted & \\
\hline & & 1630 & 6.7 & Slightly melted & \\
\hline
\end{tabular}


Table III. Compositions, Firing Conditions, Melting Behaviors, and Crystal Phase Analyses in the System YAG- $\mathrm{Y}_{2} \mathrm{Si}_{2} \mathrm{O}_{7}$

\begin{tabular}{llllll}
\hline (21) & $50: 50$ & 1650 & 1.7 & Melted & \\
& & 1600 & 0.0 & Melted (bubbles) & \\
& & 1550 & 0.3 & Melted & \\
& & 1530 & 0.4 & Slightly melted & \\
(22) & $40: 60$ & 1650 & 0.7 & Melted & \\
& & 1600 & 0.0 & Melted & \\
& & 1550 & 0.3 & Melted & G, YS \\
& & 1530 & 1.0 & Melted & \\
(23) & $30: 70$ & 1500 & 0.9 & No melting & \\
& & 1650 & 2.5 & Melted & Melted \\
& & 1500 & 0.0 & Melted & YS, G \\
\hline
\end{tabular}


Fig. 2. The system $\mathrm{Si}_{3} \mathrm{~N}_{4}-\mathrm{Y}_{2} \mathrm{Si}_{2} \mathrm{O}_{7}$.



Fig. 3. The system $\mathrm{Si}_{3} \mathrm{~N}_{4}-\mathrm{Y}_{2} \mathrm{Si}_{2} \mathrm{O}_{7}-\mathrm{Y}_{3} \mathrm{Al}_{5} \mathrm{O}_{12}$ at $1650^{\circ} \mathrm{C}$.

Figure 3 shows the $1650^{\circ} \mathrm{C}$ isothermal section of this ternary system. An extended liquid area near the oxide side (far from $\mathrm{Si}_{3} \mathrm{~N}_{4}$ ) was observed. The lowest melting composition contained $10 \mathrm{wt} \% \mathrm{Si}_{3} \mathrm{~N}_{4}, 27 \mathrm{wt} \% \mathrm{Y}_{3} \mathrm{Al}_{5} \mathrm{O}_{12}$, and $63 \mathrm{wt} \% \mathrm{Y}_{2} \mathrm{Si}_{2} \mathrm{O}_{7}$ with a ternary eutectic temperature at $1430^{\circ} \mathrm{C}$.

Higher weight losses were observed at $1650^{\circ} \mathrm{C}$. Melted samples with compositions within the liquid area often contained bubbles. A translucent nitrogen-containing glass without bubbles could be obtained only for the compositions containing $<12$ at. \% or $<6$ wt $\%$ nitrogen, compositions surrounding the lowest melting point.

\section{Summary}

The melting behaviors in the system $\mathrm{Si}_{3} \mathrm{~N}_{4}-\mathrm{Y}_{3} \mathrm{Al}_{5} \mathrm{O}_{12}-$ $\mathrm{Y}_{2} \mathrm{Si}_{2} \mathrm{O}_{7}$ were determined under $1 \mathrm{MPa}$ of nitrogen. The behavior phase diagrams of the ternary and their binaries have been presented. The ternary eutectic composition contains $10 \mathrm{wt} \%$ $\mathrm{Si}_{3} \mathrm{~N}_{4}, 27 \mathrm{wt} \% \mathrm{Y}_{3} \mathrm{Al}_{5} \mathrm{O}_{12}$, and $63 \mathrm{wt} \% \mathrm{Y}_{2} \mathrm{Si}_{2} \mathrm{O}_{7}$ with a eutectic temperature of $1430^{\circ} \mathrm{C}$.

The binary eutectic compositions and temperatures are 25 $\mathrm{wt} \% \mathrm{Si}_{3} \mathrm{~N}_{4}$ and $75 \mathrm{wt} \% \mathrm{Y}_{3} \mathrm{Al}_{5} \mathrm{O}_{12}$ at $1650^{\circ} \mathrm{C}, 10 \mathrm{wt} \% \mathrm{Si}_{3} \mathrm{~N}_{4}$ and $90 \mathrm{wt} \% \mathrm{Y}_{2} \mathrm{Si}_{2} \mathrm{O}_{7}$ at $1570^{\circ} \mathrm{C}$, and $35 \mathrm{wt} \% \mathrm{Y}_{3} \mathrm{Al}_{5} \mathrm{O}_{12}$ and $65 \mathrm{wt} \%$ $\mathrm{Y}_{2} \mathrm{Si}_{2} \mathrm{O}_{7}$ at $1520^{\circ} \mathrm{C}$. 
Table IV. Compositions, Firing Conditions, Melting Behaviors, and Crystal Phase Analyses in the System $\mathrm{Si}_{3} \mathrm{~N}_{4}-\mathrm{YAG}-\mathrm{Y}_{2} \mathrm{Si}_{2} \mathrm{O}_{7}$

\begin{tabular}{|c|c|c|c|c|c|}
\hline & $\begin{array}{l}\mathrm{Si}_{3} \mathrm{~N}_{4}: \mathrm{YAG}_{\mathrm{r}}: \mathrm{Y}_{2} \mathrm{Si}_{2} \mathrm{O}_{7} \\
\text { composition }(\mathrm{wt} \%)\end{array}$ & $\begin{array}{l}\text { Termp } \\
\left({ }^{\circ} \mathrm{C}\right)\end{array}$ & $\begin{array}{l}\text { Wt loss } \\
(w 1 \%)\end{array}$ & Melting behaviors & Phase analyses* \\
\hline$(24)$ & $80: 15: 5$ & 1650 & 0.4 & Densified & $\mathrm{SN}, \mathrm{G}, \mathrm{YS}, \mathrm{H}$ \\
\hline \multirow[t]{2}{*}{$(25)$} & $80: 10: 10$ & 1650 & 1.0 & Densified & $\mathrm{SN}, \mathrm{YS}, \mathrm{H}$ \\
\hline & & 1550 & 0.6 & No melting & \\
\hline \multirow[t]{2}{*}{$(26)$} & $80: 5: 15$ & 1650 & 1.2 & Densified & SN, YS \\
\hline & & 1550 & 0.9 & No melting & \\
\hline$(27)$ & $50: 40: 10$ & 1650 & 1.9 & Partly melted & \\
\hline$(28)$ & $50: 30: 20$ & 1650 & 2.5 & Partly melted & \\
\hline$(29)$ & $50: 20: 30$ & 1650 & 3.0 & Partly melted & \\
\hline$(30)$ & $50: 10: 40$ & 1650 & 3.9 & Partly melted & \\
\hline (31) & $40: 48: 12$ & 1650 & 1.8 & Mostly melted & \\
\hline (32) & $40: 36: 24$ & 1650 & 1.8 & Mostly melted & \\
\hline$(33)$ & $40: 24: 36$ & 1650 & 2.5 & Mostly melted & \\
\hline (34) & $40: 12: 48$ & 1650 & 3.1 & Mostly melted & \\
\hline$(35)$ & $35: 45.5: 19.5$ & 1650 & 2.6 & Melted & \\
\hline \multirow[t]{2}{*}{$(36)$} & $35: 32.5: 32.5$ & 1650 & 2.5 & Melted & \\
\hline & & 1550 & 0.8 & Mostly melted & \\
\hline \multirow[t]{2}{*}{$(37)$} & $35: 19.5: 45.5$ & 1650 & 5.0 & Melted & \\
\hline & & 1550 & 0.8 & Mostly melted & \\
\hline \multirow[t]{2}{*}{$(38)$} & $30: 56: 14$ & 1650 & & Mostly melted & \\
\hline & & 1550 & 1.8 & No melting & \\
\hline \multirow[t]{2}{*}{$(39)$} & $30: 42: 28$ & 1650 & & Melted (bubbles) & \\
\hline & & 1550 & 2.1 & Mostly melted & \\
\hline \multirow[t]{3}{*}{$(40)$} & $30: 28: 42$ & 1650 & & Melted (bubbles) & \\
\hline & & 1550 & 2.3 & Melted & \\
\hline & & 1500 & 1.3 & Partly melted & \\
\hline \multirow[t]{3}{*}{$(41)$} & $30: 14: 56$ & 1650 & & Melted (bubbles) & \\
\hline & & 1550 & 1.3 & Melted & \\
\hline & & 1500 & 1.5 & Partly melted & \\
\hline \multirow[t]{2}{*}{$(42)$} & $20: 72: 8$ & 1650 & 3.7 & Melted (bubbles) & \\
\hline & & 1600 & 0.6 & No melting & $\mathrm{G}, \mathrm{SN}, \mathrm{H}$ \\
\hline \multirow[t]{2}{*}{$(43)$} & $20: 64: 16$ & 1650 & 3.0 & Melted (bubbles) & \\
\hline & & 1550 & 0.6 & Little melted & \\
\hline \multirow[t]{2}{*}{$(44)$} & $20: 48: 32$ & 1650 & 4.8 & Mostly melted & \\
\hline & & 1550 & 1.1 & Partly melted & \\
\hline \multirow[t]{4}{*}{$(45)$} & $20: 32: 48$ & 1650 & 3.4 & Melted (bubbles) & \\
\hline & & 1550 & 0.6 & Melted & \\
\hline & & 1500 & 0.6 & Mostly melted & \\
\hline & & 1450 & 0.4 & Mostly melted & \\
\hline \multirow[t]{4}{*}{$(46)$} & $20: 16: 64$ & 1650 & 6.2 & Melted (bubbles) & \\
\hline & & 1550 & 1.2 & Mostly melted & \\
\hline & & 1500 & 0.4 & Mostly melted & \\
\hline & & 1450 & 0.1 & No melting & $\mathrm{H}, \mathrm{SN}$ \\
\hline \multirow[t]{2}{*}{$(47)$} & $15: 42.5: 42.5$ & 1550 & 0.6 & Melted & \\
\hline & & 1430 & 0.6 & No melting & \\
\hline \multirow[t]{3}{*}{$(48)$} & $15: 25.5: 59.5$ & 1550 & 2.5 & Melted & \\
\hline & & 1450 & 0.5 & Melted & \\
\hline & & 1430 & 1.2 & Mostly melted & \\
\hline$(49)$ & $15: 8.5: 76.5$ & 1650 & 9.6 & Melted (bubbles) & \\
\hline$(50)$ & $10: 72: 18$ & 1650 & 3.3 & Melted (bubbles) & \\
\hline & & 1550 & 1.4 & Partly melted & \\
\hline$(51)$ & $10: 54: 36$ & 1550 & 1.6 & Mosily melted & \\
\hline$(52)$ & $10: 36: 54$ & 1550 & 1.5 & Melted & \\
\hline & & 1500 & 1.4 & Melted & \\
\hline & & 1450 & 0.4 & Melted & \\
\hline & & 1430 & 0.1 & Little melted & \\
\hline (53) & $10: 27: 63$ & 1430 & 1.5 & Melted $\left(\mathrm{T}_{\mathrm{eu}}\right)$ & \\
\hline$(54)$ & $10: 18: 72$ & 1550 & 0.8 & Melted & \\
\hline & & 1500 & 1.6 & Melted & \\
\hline & & 1450 & 0.3 & Mostly melted & \\
\hline & & 1430 & 1.0 & Slightly melted & \\
\hline$(55)$ & $5: 28.5: 66.5$ & 1430 & & Mostly melted & \\
\hline
\end{tabular}

${ }^{*} \mathrm{SN}=\mathrm{Si}_{3} \mathrm{~N}_{4}\left(\alpha\right.$ and $/$ or $\beta$ ), G $=\mathrm{Y}_{3} \mathrm{Al}_{5} \mathrm{O}_{12}$ (YAG or garnet), $\mathrm{H}=\mathrm{Y}_{5}\left(\mathrm{SiO}_{4}\right)_{3} \mathrm{~N}\left(\mathrm{H}\right.$-phase), $\mathrm{YS}=\mathrm{Y}_{2} \mathrm{Si}_{2} \mathrm{O}_{7}$.

\section{References}

'L. J. Gauckler, H. L. Lukas, and G. Petzow, "Contribution to the Phase Diagram $\mathrm{Si}_{3} \mathrm{~N}_{4}-\mathrm{AlN}-\mathrm{Al}_{2} \mathrm{O}_{3}-\mathrm{SiO}_{2}, " J$. Aml. Ceram. Soc, 58 [7-8] 346-47 (1975).

'L. J. Gauckler. H. Hohnke, and T.-Y. Tien, "The System $\mathrm{Si}_{3} \mathrm{~N}_{1}-\mathrm{SiO}_{2}-\mathrm{Y}_{2} \mathrm{O}_{3}$," f. Am. Coram. Soc., 63|1-2| 35-37 (1980).

'I. K. Naik and T.-Y. Tien, "Subsolidus Phase Relations in Part of the System Si,Al,Y/N,O,"J. Am. Ceram. Soc., 62 [1 1-12] 642-43 (1979).

${ }^{4}$ I. K. Naik, L. J. Gauckler, and T.-Y. Tien, "Solid-Liquid Equilibrium in the System $\mathrm{Si}_{2} \mathrm{~N}_{4}-\mathrm{AIN}-\mathrm{SiO}_{2}-\mathrm{Al}_{2} \mathrm{O}_{3}$," J. Am. Ceram. Soc, 61 [7-8] 332-35 (1978).

"W.-Y. Sun, T.-Y. Tien, and T.-S. Yen, "Subsolidus Phase Relationships in
Part of the System Si,Al,Y/N,O: The System $\mathrm{Si}_{3} \mathrm{~N}_{4}-\mathrm{AlN}-\mathrm{YN}-\mathrm{Al}_{2} \mathrm{O}_{3}-\mathrm{Y}_{2} \mathrm{O}_{3}$," J. Am. Ceram. Soc, 74 [11] 2753-58 (1991).

"M. H. Lewis, A. R. Bhatti, R. J. Lumby, and B. North, "The Microstructure of Sintered Si-Al-O-N Ceramics," J. Mater. Sci, 15 |1| 103 (1980).

${ }^{7}$ H. Hohnke and T.-Y. Tien, "Solid-Liquid Reaction in Part of the System Si,Y/N,O"; pp. 101-10 in Progress in Niwogen Ceramics. Edited by F. L. Riley. Martinus Ni hoff. Boston. MA, 1983.

${ }^{x} \mathrm{M}$. Wisnudel and T.-Y. Tien, "Solid-Liquid Reactions in the System $\mathrm{Si}_{3} \mathrm{~N}_{4}-$ $\beta$-SiAlON-YAG," J. Am. Ceram. Soc., in press.

${ }^{9} \mathrm{E}$. Underwood, Quantitative Stereology. Addison-Wcsley, Reading, MA 1970 . 